\title{
A Novel Isoform of Acetylcholinesterase Exacerbates Photoreceptors Death after Photic Stress
}

\author{
Rinat Kebat, ${ }^{1,2}$ Esther Zemel, ${ }^{1}$ Nicolas Cuenca, ${ }^{3}$ Tama Evron, ${ }^{4}$ Debra Toiber, ${ }^{4}$ \\ Anat Loewenstein, ${ }^{5}$ Hermona Soreq, ${ }^{*, 4,6}$ and Ido Perlman ${ }^{*, 1,6}$
}

Purpose. To study the involvement of stress-induced acetylcholinesterase (AChE) expression in light-induced retinal damage in albino rats.

Methods. Adult albino rats were exposed for 24 hours to bright, damaging light. AChE expression was monitored by in situ hybridization, by histochemistry for AChE activity, and by immunocytochemistry. An orphan antisense agent (Monarsen; Ester Neurosciences, Ltd., Herzlia Pituach, Israel) was administered intraperitoneally to minimize light-induced AChE expression. The electroretinogram (ERG) was recorded to assess retinal function.

Results. Twenty-four-hour exposure to bright light caused severe reduction in the ERG responses and augmented expression of mRNA for the "read-through" variant of AChE (AChE-R) in photoreceptor inner segments (IS), bipolar cells, and ganglion cells. AChE activity increased in IS. The expressed AChE protein was a novel variant, characterized by an extended $\mathrm{N}$ terminus (N-AChE). Systemic administration of the orphan antisense agent, Monarsen, reduced the photic induction of mRNA for AChE-R, and of the N-AChE protein. Rats exposed to bright, damaging light and treated daily with Monarsen exhibited larger ERG responses, relatively thicker outer nuclear layer (ONL), and more ONL nuclei than did rats exposed to the same damaging light but treated daily with saline.

Conclusions. The findings indicate that the photic-induced novel variant of AChE (N-AChE-R) may be causally involved with retinal light damage and suggest the use of RNA targeting for limiting such damage. (Invest Ophthalmol Vis Sci. 2007;48: 1290 -1297) DOI:10.1167/iovs.06-0847

From the ${ }^{1}$ Ruth and Bruce Rappaport Faculty of Medicine, Technion-Israel Institute of Technology and the Rappaport Institute, Haifa, Israel; the ${ }^{2}$ Department of Ophthalmology, Bnei-Zion Medical Center, Haifa, Israel; the ${ }^{3}$ Department of Biotechnology, University of Alicante, Alicante, Spain; the ${ }^{5}$ Department of Ophthalmology, Tel Aviv Medical Center, Tel Aviv, Israel; and the ${ }^{4}$ Institute of Life Sciences, The Hebrew University of Jerusalem, Israel.

${ }^{6}$ Contributed equally to the work and therefore should be considered equivalent authors.

Supported in part by the Chief Scientist, Israel Ministry of Health, the Selma Mitrani Age Related Macular Degeneration Research Fund (IP), and the European Alternative Splicing Network of Excellence Grant EURASNET LSH-2004-1.1.5-3 (HS)

Submitted for publication July 23, 2006; revised September 30 and November 10, 2006; accepted January 17, 2007.

Disclosure: R. Kehat, None; E. Zemel, None; N. Cuenca, None; T. Evron, None; D. Toiber, None; A. Loewenstein, None; H. Soreq, None; I. Perlman, None

The publication costs of this article were defrayed in part by page charge payment. This article must therefore be marked "advertisement" in accordance with 18 U.S.C. $\$ 1734$ solely to indicate this fact.

*Each of the following is a corresponding author: Ido Perlman, Ruth and Bruce Rappaport Faculty of Medicine, Technion-Israel Institute of Technology, PO Box 9649, Haifa, Israel 31096; iperlman@tx.technion.ac.il.

Hermona Soreq, Institute of Life Sciences, The Hebrew University of Jerusalem, Jerusalem, Israel 91904; soreq@cc.huji.ac.il.
Dhotoreceptor loss is the primary cause of blindness in degenerative diseases such as retinitis pigmentosa and agerelated macular degeneration. Exposure of albino rats to bright light provides an established model for photoreceptor damage and is widely used to study the mechanisms underlying stressinduced photoreceptor injury. ${ }^{1}$ The extent of retinal damage caused by exposure to light depends on numerous factors, including the intensity and color of the light and the duration of exposure, ${ }^{2}$ age ${ }^{3}$ genotype, ${ }^{4}$ ocular pigmentation, ${ }^{5}$ and body temperature. ${ }^{6}$ The detailed pathologic mechanism(s) underlying light-induced photoreceptor damage are not yet fully understood. However, a variety of growth hormones, cytokines, ${ }^{7}$ and antioxidants ${ }^{8}$ were found to provide partial protection to retinal photoreceptors from light damage, suggesting causal involvement of stress-induced processes.

The acetylcholine (ACh)-hydrolyzing enzyme, acetylcholinesterase (AChE) is notably involved in neuronal stress reactions. ${ }^{9}$ Apart from its crucial role in terminating synaptic transmission ${ }^{10}$ at cholinergic synapses in the peripheral (PNS) and central (CNS) nervous systems, AChE also plays morphogenic roles, ${ }^{11,12}$ and participates in various stress responses. ${ }^{13}$ In mice, neuronal AChE levels are markedly increased after forced swimming and exposure to anticholinesterases, ${ }^{14}$ even in brain regions that do not primarily participate in cholinergic activity. Stress-induced expression of AChE is accompanied by a prominent shift in the protein C terminus from the "synaptic" variant to the "read-through" sequence, ${ }^{15}$ both including the core domain required for hydrolyzing $\mathrm{ACh},{ }^{9}$ as described schematically in Figure 1. Alternate promoter usage produces each of these AChE variants in two versions, differing in the length of their N-terminal domain. ${ }^{16}$ Thus, AChE-R can appear either with a cleavable $\mathrm{N}$-terminal signal peptide or with an extended $\mathrm{N}$ terminus, potentially making it membrane bound (Fig. 1c). However, the functional role(s) of the extended N-AChE proteins under stressful insults remained to be determined.

The stress-related role of neural AChE was inferred from experiments in which overexpression of AChE-R mRNA was suppressed in mice subjected to closed head injury by administration of Monarsen, an orphan drug antisense oligonucleotide. ${ }^{17}$ Monarsen reduced the number of dead neurons, and facilitated neurologic recovery, suggesting that the R-variant(s) of AChE and/or secondary element(s) induced by overexpression of AChE-R contributed to neuronal death. This could reflect a direct contribution of the increased hydrolytic capacity of the induced AChE to reduce the tissue level of acetylcholine, promoting inflammation and tissue damage. ${ }^{18}$ Alternatively, or in addition, stress-induced AChE could selectively contribute to cellular apoptosis through its noncatalytic properties, compatible with reports of others. ${ }^{19,20}$ In that case, excess AChE could induce cell death in noncholinergic neurons, as well.

The retina, as part of the central nervous system (CNS), may also depend on morphogenic roles of AChE during development and after stress episodes. The importance of AChE gene expression for retinal development was experimentally validated in a detailed neuroanatomic survey that demonstrated impaired formation of the inner retina and degeneration of photoreceptors in an AChE knockout mouse. ${ }^{21}$ However, 


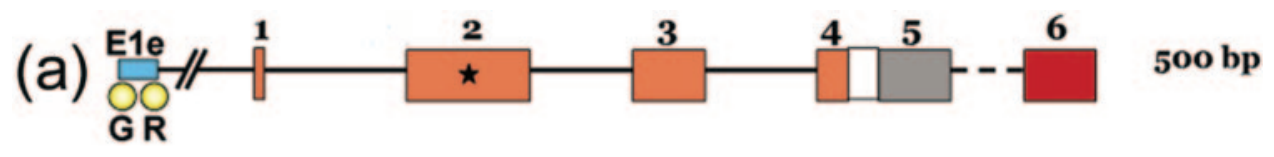

(b)

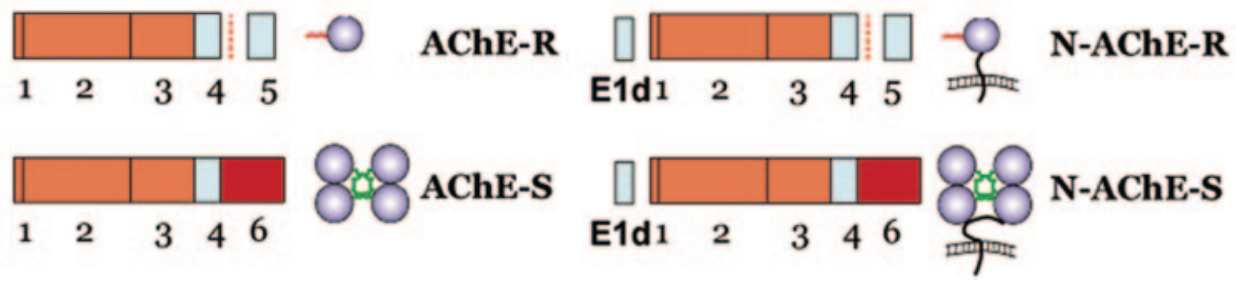

FIGURE 1. Schematic representation of the mouse AChE gene and its mRNA variants and proteins. (a) Schematic representation of the AChE gene (exons are shown as boxes and introns as lines). There are five alternative versions of exon 1 (E1a-E1e) in the mouse AChE gene (only the last one is shown). (b) AChE-mRNA variants may differ due to 3 ' splicing. The "synaptic" protein product (AChE-S) is membrane bound and is associated with cholinergic synapses, whereas the "read-through" protein (AChE-R) is induced by stress in a secreted form. Each of these mRNA variants can appear with a short, conservative (b) or an extended $\mathrm{N}$ terminus (c). It is believed that the proteins with the extended $\mathrm{N}$ terminus have additional means to be anchored to membranes. GR, glucocorticoid response element.

these findings are relevant to the embryonic development of the retina and not to stress-induced malfunction in the adult retina. We have shown AChE mRNA expression in human adult photoreceptors $^{22}$ - cells that are not involved in cholinergic synaptic activity - raising the possibility that AChE in photoreceptors exerts stress-related morphogenic function(s). This hypothesis is particularly appealing, since the major excitatory neurotransmitter of the retina is L-glutamate, ${ }^{23}$ whereas ACh plays a limited role in visual information processing within the retina and is found mainly in amacrine and ganglion cells in the proximal part of the retina. ${ }^{24,25}$

We tested the hypothesis that exposure to damaging light is a stressful event that induces the expression of AChE in the retina, with particular interest in the photoreceptors. We further identified the nature of the induced AChE variant and its role in retinal damage.

\section{Methods}

\section{Procedure}

Adult male Sprague-Dawley albino rats $(N=60)$, weighing 200 to $300 \mathrm{~g}$, were kept for at least 2 weeks in a 12-hour light-dark cycle with free access to food and water. Rats were exposed for 24 hours, starting at around $10 \mathrm{AM}$, at room temperature, to bright white light (260 foot-candles) in a specially designed light chamber, and then returned to the normal rearing conditions (12-hour light-dark cycle). To assess retinal function, we recorded the electroretinogram (ERG). For ERG recordings, rats were anesthetized by an intramuscular injection of ketamine hydrochloride $(3.3 \mathrm{mg} / \mathrm{kg})$, acepromazine maleate $(6.7 \mu \mathrm{g} /$ $\mathrm{kg})$, and xylazine $(2 \mu \mathrm{g} / \mathrm{kg})$. At the termination of the ERG follow-up period, rats were killed by an overdose of sodium pentobarbital, injected intraperitoneally, and their retinas were prepared for in situ hybridization (ISH), histochemical evaluation or immunocytochemistry. Eyes were enucleated and incubated (1-hour for cryosections and 24-hour for paraffin sections) in $0.1 \mathrm{M}$ phosphate buffer ( $\mathrm{pH} 7.4$ ) containing $4 \%$ paraformaldehyde $\left(4^{\circ} \mathrm{C}\right)$.

The rats were treated in compliance with the ARVO Statement for the Use of Animals in Ophthalmic and Vision Research and in accordance with institutional guidelines.

\section{Immunocytochemistry}

Cryostat sections were incubated overnight, under agitation, with affinity-purified primary rabbit antiserum targeted against a synthetic peptide unique for rodent $\mathrm{N}-\mathrm{AChE}^{16}$ at a dilution of 1:100 in 0.1 M PBS $1 \%$ Triton X-100. We also used an antibody for the core domain, common to all AChE variants (N-19; Santa Cruz Biotechnology, Santa Cruz, CA). Secondary antibodies were donkey anti-rabbit IgG coupled to fluorescein isothiocyanate (FITC) at a 1:100 dilution and a donkey anti-goat IgG coupled to $\mathrm{Cy} 3$ at a 1:100 dilution. Slides were viewed using a confocal microscope (Eclipse E600; Nikon, Tokyo, Japan; and Radiance 2000; Bio-Rad, Hercules, CA) scanning system.

\section{Histochemical Staining for AChE Activity}

Cryostat sections were stained for catalytically active AChE according to Karnovsky and Roots. ${ }^{26}$ Overnight incubation was done in a medium containing freshly prepared acetylthiocholine iodide (AThCh) as a substrate in $0.1 \mathrm{M}$ phosphate buffer $(\mathrm{pH} 6.0 ; 0.8 \mathrm{mg} / \mathrm{mL}), 0.1 \mathrm{M}$ sodium citrate, $30 \mathrm{mM}$ copper sulfate, distilled deionized water (DDW), and 5 $\mathrm{mM}$ potassium ferricyanide. The BChE inhibitor, iso-OMPA (tetraisopropylpyrophosphoramide; Sigma-Aldrich, St. Louis, MO) was added $\left(10^{-5} \mathrm{M}\right)$ to specifically localize AChE activity. The selective AChE inhibitor BW 284C51 $\left(10^{-5} \mathrm{M}\right)$ was used as a negative control. Brownish staining, resulting from AThCh hydrolysis, was examined under a light microscope (BH2; Olympus, Tokyo, Japan).

\section{In Situ Hybridization}

ISH was performed on paraffin-embedded sections, $5 \mu \mathrm{m}$ thick, as previously described. ${ }^{13}$ Hybridization involved overnight incubation at $52^{\circ} \mathrm{C}$ in hybridization mix with a $5^{\prime}$-biotinylated, $2^{\prime}$-O-methylated $\mathrm{AChE}$ mRNA probe complementary to either the AChE-S (exon 6) rat isoform: (5402) 5'-CCGGGGGACGUCGGGGUGGGGUGGGGA UGGGCAGAGUCUGGGGCUCGUCU-3' (5352); or the AChE-R (intron 4) rat isoform: (4397) 5'-CUAGGGGGAGAAGAGAGGGGUUACAC UGGCGGGCUCCCACUCCCCUCCUC-3' (4349). The numbers in parentheses denote nucleotide positions in the GenBank-deposited sequence (accession no. M55040; http://www.ncbi.nlm.nih.gov/Genbank; provided in the public domain by the National Center for Biotechnology Information, Bethesda, MD).

\section{Analysis}

For quantitative analysis of staining in the ISH and histochemistry tests, we photographed slides and converted the micrograph to grayscale. Densities of retinal layers were quantified in arbitrary units by densitometry (TINA software ver. 2.10; Raytest, Straubenhardt, Germany). In each slide, we chose a rectangular window of width smaller than that of the narrowest layer to be measured, the photoreceptor inner 
segments (IS). The same window was used to measure density of the different retinal layers. Four random measurements were made in each retinal layer of a given retinal micrograph and averaged. The density of the outer nuclear layer (ONL) was set as the background, and all density measurements from other retinal layers were performed relative to this background. In this manner, we ensured that density measurements would be independent of technical factors such as thickness of section, duration of incubation, angle of slicing and others that may affect absolute density measurements but not relative measurements. Data from retinas of four rats undergoing the same experimental procedure were averaged. Because of the small sample size ( $n$ $=4$ ), densitometry measurements were compared for statistical significance by using the Mann-Whitney nonparametric test.

\section{Antisense Treatment}

Monarsen (Ester Neurosciences, Ltd., Herzlia Pituach, Israel) antisense oligodeoxynucleotide directed against Rat AChE mRNA, a 20-mer oligodeoxynucleotide ( $5^{\prime}$-CTGCAATATTTTCTTGCACC- ${ }^{\prime}$ ) complementary to a sequence in exon 2 of rat AChE mRNA, was used as previously described. ${ }^{27}$ A single dose of Monarsen was found to have a transient action lasting between 24 and 72 hours, depending on the injected dose. ${ }^{27}$ Since development of light-induced retinal damage proceeds at a relatively slow rate, we decided to inject Monarsen on a daily basis throughout the entire duration of the follow-up (30 days). Intravitreal injection, the preferred route for retinal treatment, was an unrealistic solution for the treatment. Because the blood-retinal barrier had been shown to be disrupted after exposure to bright light, ${ }^{28}$ we decided to inject Monarsen intraperitoneally. Experimental rats were treated for 30 days with daily intraperitoneal injections of Monarsen $(0.1 \mathrm{~mL}$ at a dosage regimen of $500 \mu \mathrm{g} / \mathrm{kg}$ ), beginning from 1 day before light exposure, while rats in the control group were injected intraperitoneally each day with an equal volume of saline.

\section{Electroretinogram}

The $\mathrm{ERG}^{29}$ is the light-induced electrical activity of the retina that is used to assess retinal function. ${ }^{30}$ It is composed of two major components; the negative a-wave reflects light-induced electrical activity in the photoreceptors, whereas the positive b-wave is generated in postreceptor retinal neurons, mainly ON center bipolar cells. ${ }^{31,32}$

Rats, kept overnight in total darkness, were anesthetized, as described earlier. The pupils were dilated (cyclopentolate hydrochloride $1 \%$ ), and topical anesthetic drops (benoxinate $\mathrm{HCl} 0.4 \%$ ) were used to assure the animal's comfort. A heating pad was used to maintain body temperature at $37^{\circ} \mathrm{C}$. ERG responses were recorded simultaneously from both eyes using corneal electrodes (Medical Workshop, Groningen, The Netherlands). Light stimuli were obtained from a Ganzfeld light source (LKC Technologies, Gaithersburg, MD) with a maximum intensity of $5.76 \mathrm{~cd}-\mathrm{s} / \mathrm{m}^{2}$

The amplitude of the ERG a-wave was measured from baseline to the trough of the wave, whereas the amplitude of the ERG b-wave was measured from the trough of the a-wave to the peak of the b-wave. Because the amplitude of the ERG waves depend on the intensities of the light stimuli, it is customary to assess retinal function quantitatively, by fitting the response-intensity data to a hyperbolic function ${ }^{33,34}$ :

$$
V / V_{\max }=I /(I+\sigma)
$$

where $V$ and $V_{\max }$ are the amplitudes of the ERG waves that are elicited respectively by a flash of intensity $I$ and by a flash of supersaturating intensity, and the semisaturation constant $\sigma$ is a measure of retinal sensitivity.

\section{Results}

AChE gene expression is notably subject to complex stressinduced changes (Fig. 1). To assess the effects of photic stress on expression and activity of AChE, we compared retinas of 15 albino rats, exposed to bright, damaging light for 24 hours to those of 15 unexposed control albino rats, raised in a 12-hour light-dark cycle. After exposure to the bright, damaging light, all 30 rats were kept in a regular 12-hour light-dark cycle with no further treatment for the entire 30-days of follow-up (experiment 1). Retinal micrographs from rats participating in this experiment clearly demonstrated light-induced modifications in AChE expression and activity (Fig. 2). In the control retina, ISH (Fig. 2a) predictably indicated very low levels of expression of mRNA for the AChE-R variant. After exposure to damaging light, expression of AChE-R mRNA was augmented in the ganglion cells (GCs) and in retinal cells in the INL. However, the most striking effect was seen in the inner segments (IS) of the photoreceptors. This effect was noted as early as 1-day after exposure to damaging light, increased during the next 14 days (Figs. 2c, 2e, arrowheads), and was seen up to 30 days (data not shown), the longest period of follow-up. Densitometry measurements showed a mild, but statistically significant, increase in IS AChE-R mRNA at 1 day after exposure, and a substantial, statistically significant, increase at 14 days after exposure to light (Table 1). Expression of AChE-S mRNA, encoding the synaptic variant of AChE was relatively unchanged after light exposure, compared with normal rat retinas (data not shown).

The augmented expression of AChE-R mRNA was accompanied by increased cytochemical labeling for total AChE catalytic activity (Fig. 2, right column), albeit at a different pattern. In the control retina, strong AChE activity was seen in the proximal parts of the retina (IPL and ganglion cells) and very weak activity in the photoreceptor inner segments (Fig. 2b), reflecting the normal activity of the AChE-S variant in the rat retina. ${ }^{24,25}$ It should be noted that the staining pattern in the proximal retina was rather diffuse and lacked the distinct layered staining, ${ }^{24}$ probably because we had to incubate the slices overnight to test for catalytic activity in the photoreceptor inner segments. With shorter incubation times (2- 4 hours), the staining pattern in the proximal retina (data not shown), was very similar to that shown previously. ${ }^{24}$

At 1 day and 14 days after exposure to bright, damaging light, AChE activity increased in the photoreceptor inner segments (Figs. 2d, 2f, arrowheads; Table 1). AChE activity in the photoreceptors was refractory to iso-OMPA, an inhibitor of the AChE-homologous protein butyrylcholinesterase (BChE), but was completely blocked by BW 284C51, a selective inhibitor of AChE (data not shown), indicating that exposure to bright, damaging light increased AChE but not BChE activity in the photoreceptors. It was difficult to appreciate stress-induced changes in AChE activity in the proximal part of the retina (INL and GC), because histochemical staining was already high in the control retinas, obscuring any stress-induced augmentation. Therefore, photic stress-related changes in AChE activity were analyzed quantitatively only for the photoreceptor inner segments (IS in Table 1).

The light-exposure-induced AChE-R accumulation in the photoreceptor inner segments could be an irrelevant byproduct of the damaging stress. Alternatively, it could have a detrimental role promoting light-induced photoreceptor death, or could be part of a recovery process. To distinguish between these possibilities, rats were treated daily by intraperitoneal injection of rat Monarsen, an antisense agent directed against the consensus domain in AChE mRNA. ${ }^{27,35,36}$ Treatment initiated 1 day before light exposure and proceeded until the end of the follow-up period. Control rats in this experiment (experiment 2), were also exposed to the damaging, bright light, but were injected intraperitoneally daily with saline solution. We chose intraperitoneal injection for Monarsen administration, because daily intravitreal injection for 30 consecutive days was unrealistic, and because previous reports indicated a breakdown of the blood-retinal barrier after exposure to dam- 


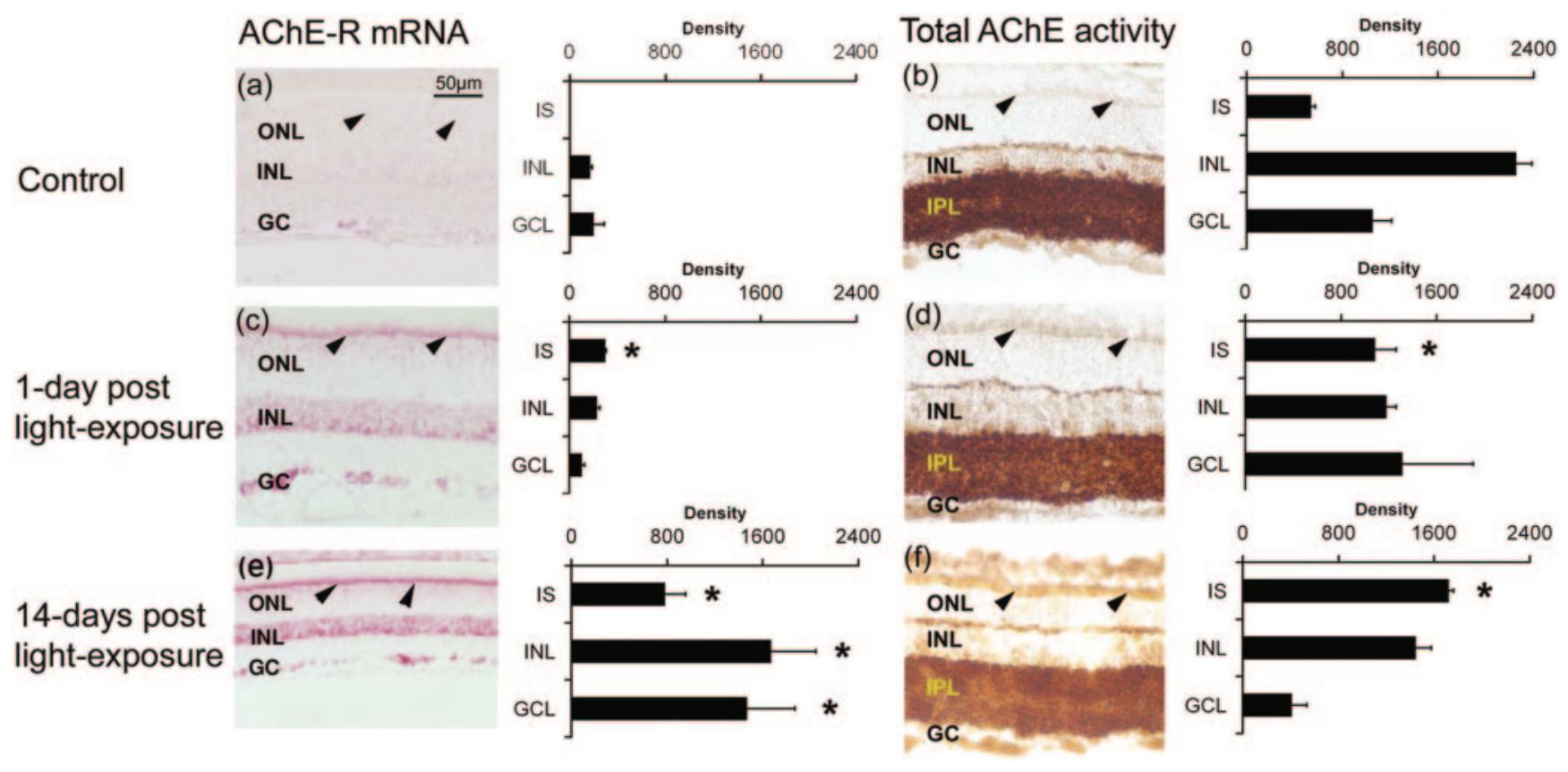

FigURE 2. Expression of AChE-R mRNA (ISH) and total AChE activity (cytochemistry) in the retinas of a control rat and of rats exposed to damaging light and killed 1 day and 14 days after exposure. Average ( \pm SEM) densities at the photoreceptors inner segments (IS), inner nuclear layer (INL), and ganglion cells (GC), obtained from retinas of four rats, are shown to the right of each representative micrograph. AChE-R mRNA was found at a very low level in control rats (a), but was significantly expressed in rats exposed to bright, damaging light (c, e). Light-induced AChE-R mRNA expression was evident in ganglion cells (GC), in neurons of the inner nuclear layer (INL), and in the inner segments (IS) of the photoreceptors (arrowheads). High AChE activity was found in the inner plexiform layer (IPL) of retinas from unexposed control rat (b) and of experimental rats exposed to damaging light (d, f). AChE activity was also evident in the inner segments of the photoreceptors (arrowheads) of rats exposed to damaging light $(\mathbf{d}, \mathbf{f})$. *Statistically significant differences between light-exposed and control rats $(P<0.05)$.

aging light, ${ }^{28}$ thus providing a simple route for delivering the antisense agent to the retina.

Figure 3 shows micrographs of retinas that were obtained 14 days after exposure to damaging light and underwent ISH for AChE-R mRNA (Figs. 3a, 3c), or histochemical staining for total AChE activity (Figs. 3b, 3d). Sections were derived from experimental rats treated with Monarsen (Figs. 3c, 3d), and from control rats treated with saline (Figs. 3a, 3b). It should be noted that in preliminary experiments, Monarsen treatment, either by intravitreal injection or by intraperitoneal injection, exerted no effects on the retinas of rats, which were raised under a 12-hour light-dark cycle and were not exposed to bright, damaging light (data not shown), similar to its null effects in the control brain. ${ }^{37}$ In contrast, averaged densitometry measurements from retinas of four rats of each group in this experiment (right of each micrograph) demonstrated that Monarsen, injected intraperitoneally, predictably crossed the blood-retinal barrier, reached the retina, and suppressed the light-induced expression of AChE-R mRNA in all retinal cells, having the strongest effect in the photoreceptor inner segments (Fig. 3c, arrowheads). Correspondingly, AChE activity in the photoreceptor inner segments was considerably weaker in the retina from rats treated with Monarsen (Fig. 3d, arrowheads) compared with those injected with saline (Fig. 3b, arrowheads). An important finding is that Monarsen did not alter AChE activity in the proximal retina (IPL and GC), sug-

TABLE 1. The Effects of 24-hour Exposure to Bright, Damaging Light upon Expression of AChE-R mRNA

\begin{tabular}{|c|c|c|c|c|c|}
\hline & \multirow[b]{2}{*}{ Control } & \multicolumn{2}{|c|}{$\begin{array}{l}\text { Experiment 1: Light } \\
\text { Exposure with No } \\
\text { Further Treatment }\end{array}$} & \multicolumn{2}{|c|}{$\begin{array}{l}\text { Experiment 2: } 14 \text { Days } \\
\text { after Light Exposure } \\
\text { with Daily Injection }\end{array}$} \\
\hline & & 1 Day & 14 Days & Saline & Monarsen \\
\hline $\mathrm{Ph}$ IS (ISH) & 1.0 & $296^{*}$ & $781^{*}$ & 1141 & $25.5 \dagger$ \\
\hline INL (ISH) & 1.0 & 1.4 & $10.2^{*}$ & 12.1 & $1.0 \dagger$ \\
\hline GC (ISH) & 1.0 & 0.5 & $7.4^{*}$ & 5.0 & 3.2 \\
\hline IS (Cytochemical staining) & 1.0 & $2.0^{*}$ & $3.2^{*}$ & 3.4 & $1.9 \dagger$ \\
\hline
\end{tabular}

Effects were measured in photoreceptors inner segments (Ph IS), inner nuclear layer (INL) and ganglion cells (GC). AChE activity was measured in photoreceptors inner segments (IS). Experiment 1: Exposure to bright, damaging light and follow-up without any treatment. Experiment 2: Exposure to bright, damaging light followed by daily intraperitoneal injection of saline or Monarsen (antisense). Values indicate fold increase of staining relative to the corresponding layer in control rats that were not exposed to the bright, damaging light. Each value is the average of measurements in retinas from four different rats undergoing the same experimental procedure.

* Statistically significant $(P<0.05)$ difference when compared to control.

† Statistically significant $(P<0.05)$ difference when compared to rats treated daily with saline. 


\section{AChE-R mRNA}

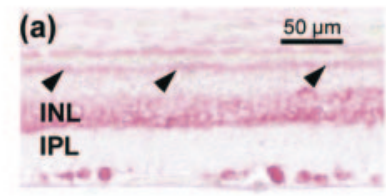

Saline

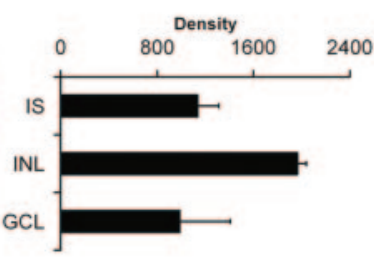

Total AChE activity
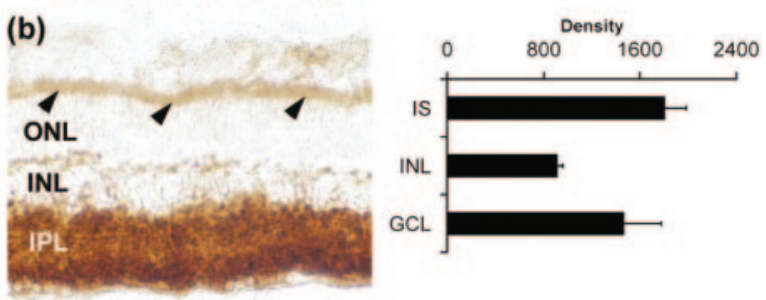

(c)

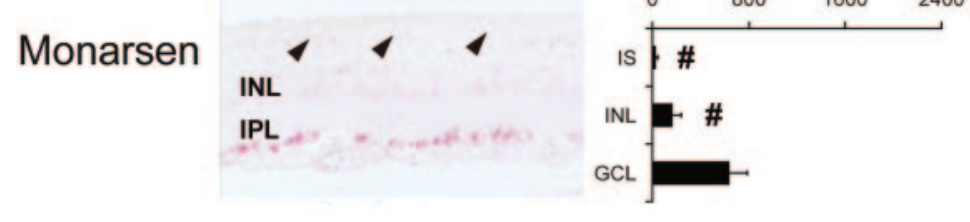

(d)

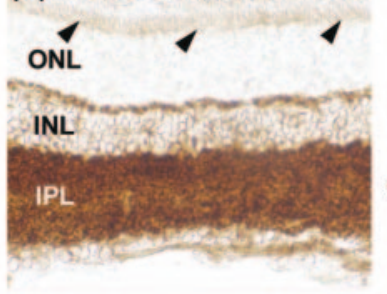

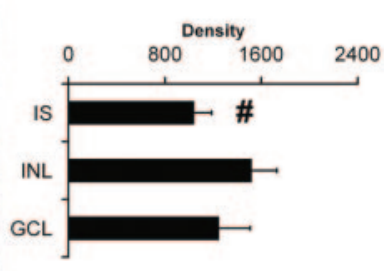

Figure 3. The effects of Monarsen, an orphan AChE mRNA-targeted antisense agent, on light-induced expression of AChE-R mRNA and total activity of AChE. Rats were treated daily with intraperitoneal injection of saline or Monarsen and were killed 14-days after exposure (24 hours) to bright, damaging light. Average ( \pm SEM) density values at the photoreceptors inner segments (IS), inner nuclear layer (INL), and ganglion cells (GC), obtained from retinas of four rats, are shown to the right of each representative micrograph. AChE-R mRNA and AChE activity were higher in the retinas of rats, exposed to bright, damaging light, and treated with saline (a, b), compared with light-exposed rats that had been treated with Monarsen (c, d). Most evident was the effect in the inner segments of the photoreceptors (arrowheads). \#Statistically significant differences between Monarsen- and saline-treated rats $(P<0.05)$.

gesting that these layers express AChE-S, which is considerably less sensitive to this agent. This supports previous observations in rat muscle, ${ }^{27}$ mouse brain, ${ }^{36}$ and primate motor neurons, ${ }^{35}$ indicating that nascent mRNA transcripts of AChE are more susceptible to the antisense treatment, probably because they are not yet covered with the protective bulky structure of the ribosomes and translational protein complexes which engulf functioning mRNA chains. Reproducible AChE-R suppression occurred in four rats treated with Monarsen compared with four control rats. Densitometry measurements were further used to calculate the effects of saline and Monarsen treatment on AChE-R mRNA expression in the different retinal layers and on AChE activity in the photoreceptor inner segments (Table 1). The suppressive effects of Monarsen on light-induced expression of AChE-R mRNA were statistically significant in photoreceptor inner segments (IS) and in neurons of the INL, but not in ganglion cells. Monarsen's effect was also statistically significant for AChE activity in photoreceptor inner segments (IS).

An important finding was that expression of AChE-R mRNA in rats exposed to bright, damaging light and injected daily with saline was higher, albeit not significantly so, than that of rats undergoing the same exposure to light without further treatment (Table 1). This observation suggests that general trauma to the rat, associated with daily handling and injection may be also associated with increased expression of retinal AChE-R.

AChE-R can appear in two forms, one with the conservative $\mathrm{N}$ terminus, ${ }^{38}$ and the other with an extended $\mathrm{N}$ terminus ${ }^{16}$ (Fig. 1). To study the composition and localization of the AChE-R protein in photic-stressed retinas, we used a specific antibody directed at the unique N-terminally extended epitope of rodent AChE. We compared retinas of control rats that were not subjected to the damaging light with those undergoing exposure to damaging light and treated with either saline or Monarsen. We also tested the retinas for all AChE isoforms, including those with the shorter, conservative $\mathrm{N}$ terminus, using a commercially available antibody targeted to the core domain common to all variants (N-19; Santa Cruz Biotechnology). The control retina was positively stained for total AChE in the proximal margin of the INL (amacrine cells), in the IPL and in ganglion cells, whereas immunostaining of N-AChE was negligible (Fig. 4, top). A similar distribution of total AChE was found in the light-exposed rats, injected with saline (Fig. 4; second row, middle column). Exposure to light also induced intense labeling for $\mathrm{N}-\mathrm{AChE}$ in the photoreceptor inner and outer segments (Fig. 4, second row, arrowheads), and in the distal portion of the INL (Fig. 4; second row, arrows). From the location of the stained cells in the INL and their structure, they appeared to be bipolar cells. Together with the ISH data (Figs. 2,3 ), these findings suggest the accumulation of N-AChE-R in the light-exposed retina. In contrast, the retinal sections from rats, exposed to damaging light but treated with Monarsen, showed an immunostaining pattern similar to that of control rats (Fig. 4, lower row). Weak staining was seen at the photoreceptor level, and in the distal margin of the INL, suggesting that Monarsen prevented most, but not all, of the light-induced expression of N-AChE protein.

To challenge the functional role of AChE-R in light-induced retinal damage, we recorded the dark-adapted ERG before and at different time intervals after exposure to bright, damaging light. The ERG is an excellent test for our goal-that is, assessment of retinal function during an extended period of followup. The ERG reflects light-induced electrical activity of the entire retina. ${ }^{30}$ It is composed mainly of two waves: the negative a-wave reflects light-induced activity in the photoreceptors, and the positive b-wave is generated in the INL, mainly by ON-bipolar cells with contribution from Müller cells. ${ }^{31,32}$ After exposure, rats were treated daily with saline $(n=10)$ or Monarsen $(n=10)$, as described before. The ERG responses of all light-exposed rats were predictably attenuated, indicating substantial light-induced retinal damage. However, Monarsentreated rats demonstrated ERG responses of considerably larger amplitude compared with saline-treated rats, as illustrated for two rats (one of each group) in Figure 5a. These responses were elicited in the dark-adapted state with the brightest light stimulus available in our photic stimulation system. Because the ERG responses depended on the light intensity of the stimulus used to elicit them, we recorded the ERG responses to light stimuli of different intensities and constructed the re- 
FIGURE 4. Light-induced stress in turn induces N-AChE production in a Monarsen-suppressible manner. Immunocytochemistry of retinas from rats exposed to bright, damaging light and treated with saline (second row) or Monarsen (third row) are compared to that of a control rat that was not exposed to damaging light (first row). Two antibodies were used, one against AChE with extended $\mathrm{N}$ terminus (left column) and the other against the core domain of all AChE variants (middle column), as indicated in the scheme above the micrographs. The two micrographs of each rat were merged to show the overlap between the two proteins (right column). Exposure to damaging light clearly increased expression of N-AChE in the saline-treated retina, which was significantly prevented by Monarsen treatment. $\mathrm{N}$-AChE expression by exposure to bright light was mainly evident in the inner and outer segments of the photoreceptors (arrowheads) and in cells located in the inner nuclear layer (arrows) as shown in the merged micrograph (middle row, right column).

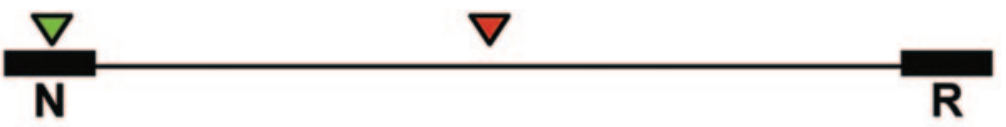

\section{$\mathrm{N}-$ Extended terminus}
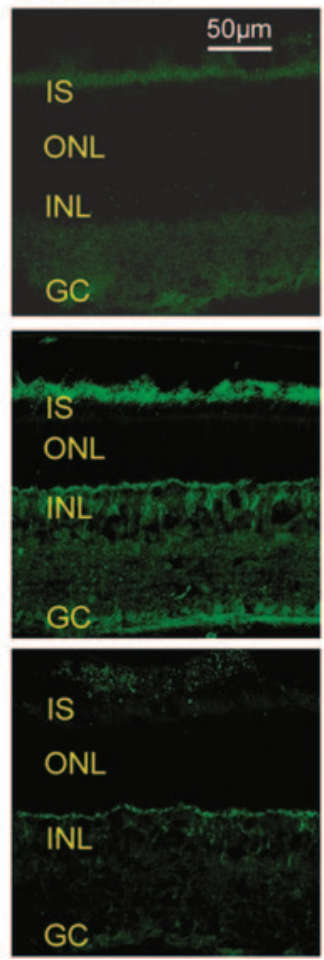

AChE core domain
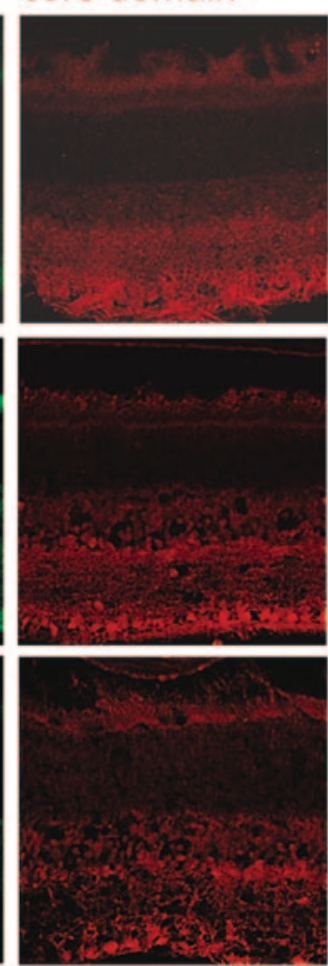

Merge
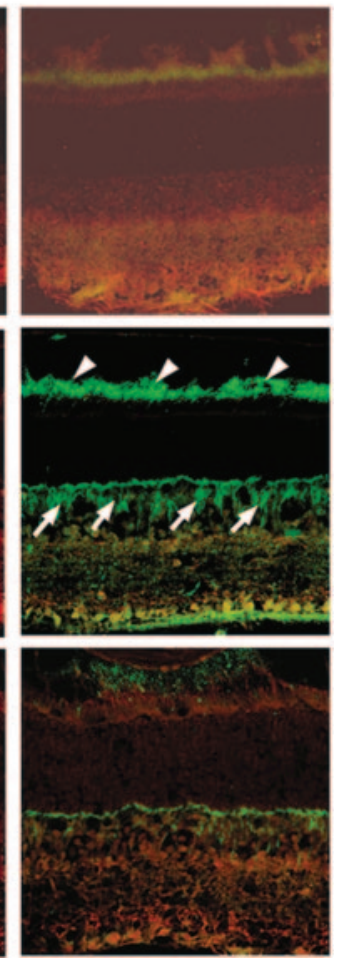

sponse-intensity curves for the a- and b-wave, as illustrated in Figures $5 b$ and $5 c$ for the two rats whose ERG responses are shown in Figure 5a. The response-intensity data were fitted to the hyperbolic function (equation 1) and the maximum response amplitude $\left(V_{\max }\right)$ of the a- and b-wave were derived.

ERG results, similar to those shown in Figure 5 were obtained from all the rats studied in this experiment. $V_{\max }$, derived from the response-intensity data, was averaged according to the two treatment groups. Monarsen-treated rats showed a lesser degree of initial ERG deficits and better recovery of the a- and b-wave compared with saline-injected rats (Fig. 6). These differences were significant for the b-wave of all the rats throughout the follow-up period $(P<0.001)$.

An additional measure, commonly used, for quantitative assessment of light-induced retinal damage, is the thickness of the ONL. Because this parameter varies considerably as a function of retinal eccentricity (distance from the optic disc), along the vertical meridian, ${ }^{7,39}$ we measured ONL thickness in the superior part of the retina $\sim 200 \mu \mathrm{m}$ from the optic disc. Measurements from three control rats (four sections in each (a) ERG Responses

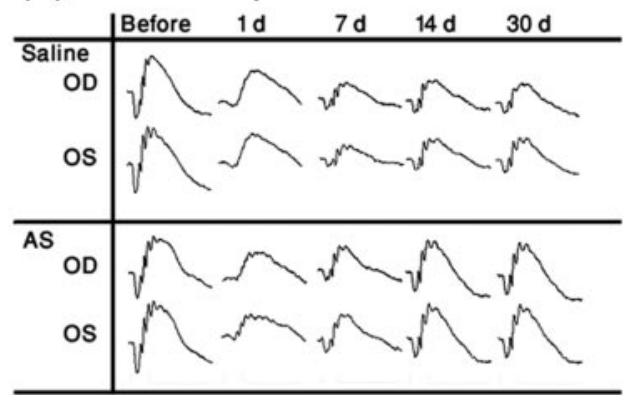

(b) Light-damage, Control

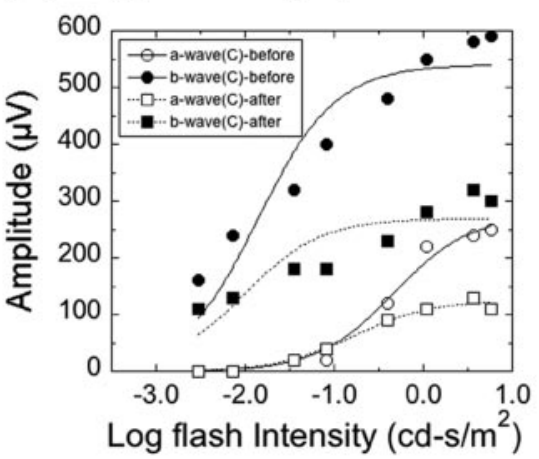

(c) Light-damage, Monarsen

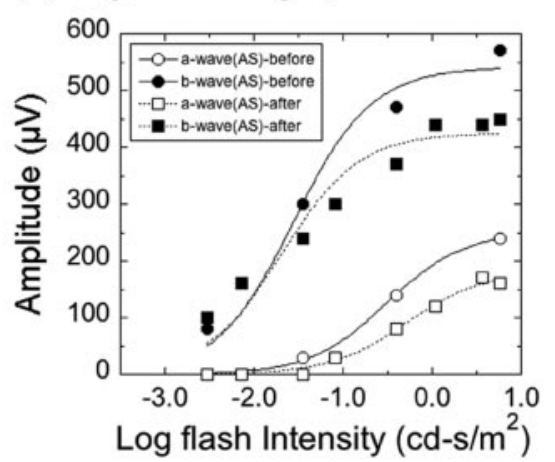

FIGURE 5. The effects of Monarsen on light-induced retinal damage, as assessed by the ERG responses. (a) ERG responses of a Monarsen-treated rat (bottom) and a saline-treated rat (top) that were recorded at different time intervals after exposure to bright, damaging light. The ERG responses were elicited from both eyes, using unattenuated bright $\left(\log I=0.76 \mathrm{~cd}-\mathrm{s} / \mathrm{m}^{2}\right)$ light stimuli. To assess the degree of light-damage and the protection by Monarsen, we constructed the response-intensity relationships of the ERG a-wave and b-wave of the right eye of the control (saline-treated) and experimental (Monarsen-treated) rats (b, c, respectively), measured before and 30-days after exposure to damaging light. The response-intensity curves were fitted to the hyperbolic function (equation 1$)$ to derive the maximum response amplitudes ( $V_{\text {max }}$ ) of the a- and b-waves. 

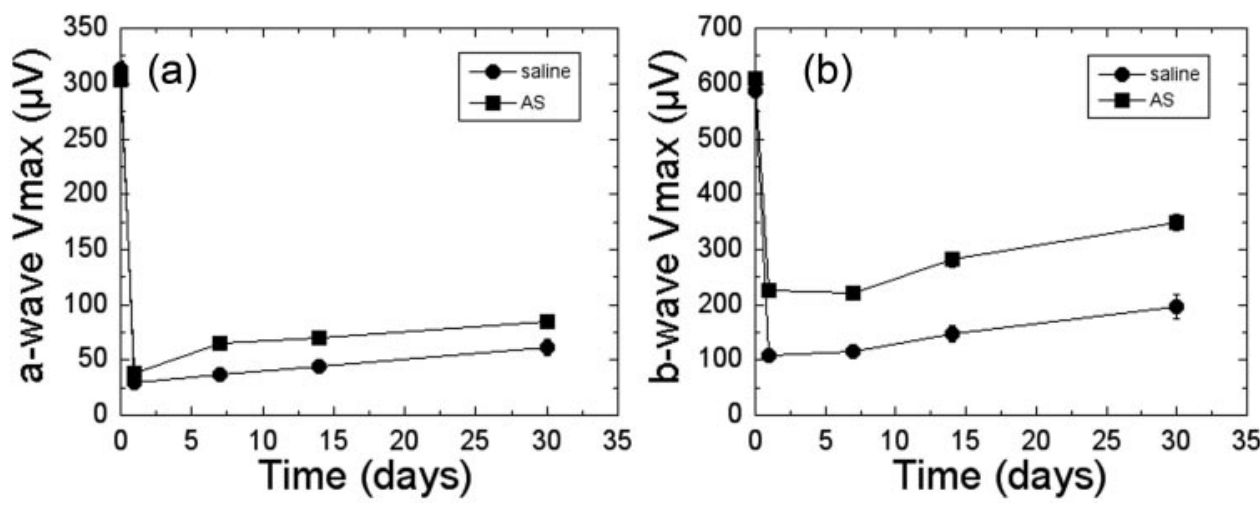

Figure 6. The effects of Monarsen on light-induced retinal damage, as assessed by the ERG responses. The maximum amplitudes of the a- and b-waves were derived from the response-intensity curves of salinetreated $(n=10)$ and Monarsentreated rats $(n=10)$. Average $( \pm \mathrm{SD})$ $V_{\max }$ compared for the ERG a-wave (a) and b-wave (b). Monarsen treatment reduced the degree of lightinduced damage, and improved recovery from photic stress. rat) that were kept in a 12-hour light-dark cycle and were not exposed to damaging light yielded an average ONL thickness of $44.1 \pm 1.7 \mu \mathrm{m}$. Similar measurements from saline-treated and Monarsen-treated rats (three rats in each group, four sections from each rat), that were prepared for histology 14 days after 24-hour exposure to bright, damaging light, gave the following average values, respectively: $22 \pm 3.0$ and $21.5 \pm 3.0$. Because the sections varied in total retinal thickness, we normalized ONL thickness to total retinal thickness. With this approach, the ONL's relative thickness in Monarsen-treated rats $(0.217 \pm$ $0.034)$ was significantly $(P<0.05)$ more than the ONL's relative thickness in saline-treated rats $(0.160 \pm 0.026)$. We also counted the number of nuclei in the ONL of these retinas by using a rectangle of $115 \mu \mathrm{m}$ width and height that was slightly larger than the width of the ONL. We chose this approach instead of counting the number of nuclei rows, because we noticed multiple loci of missing nuclei spread in a mosaic pattern within the ONL of light-damaged retinas. We measured $103.3 \pm 10.3$ nuclei in control rats not exposed to the damaging bright light. This was significantly $(P<0.001)$ larger than the number of nuclei in light-damaged retinas regardless of the treatment that was given to the rats. The number of ONL nuclei in the Monarsen-treated rats (43.8 \pm 7.6) was significantly $(P=0.009)$ larger than the number of ONL nuclei in the saline-treated rats $(31.8 \pm 4.4)$. Thus, the protective role of Monarsen against light-induced retinal damage was also evident from morphometric measurements.

\section{Discussion}

Our findings demonstrate, for the first time, that a "readthrough" isoform of AChE with an extended $\mathrm{N}$ terminus is induced by stress in the mammalian retina. The N-AChE protein(s) was also observed in other stress-responding parts of the $\mathrm{CNS}^{16}$; however, our current findings provide the first direct evidence on both its stress-induced accumulation in retinal photoreceptors and its causal involvement in the lightinduced death of these cells. This conclusion is based on two observations. ISH indicated that photic stress induced the expression of mRNA coding for the "read-through" variant of AChE, whereas immunostaining indicated expression of a protein isoform of AChE carrying an extended $\mathrm{N}$ terminus. Together, these findings suggest that the N-terminally extended AChE-R variant (N-AChE-R protein) is expressed in the retinas of albino rats subjected to photic stress. However, we could not completely rule out the possibility that N-AChE-S was also induced by exposure to bright light. We showed that this novel isoform of AChE exerted antisense-suppressible neuronal cell damage, as assessed from functional tests (ERG recordings) and morphometric measurements in the albino rat retina.

In the photic-stressed rats, Monarsen antisense treatment effectively suppressed the expression of N-AChE (Fig. 4) in the retina, while enhancing the recovery of treated retinas as evident from functional tests (Figs. 5, 6) and from morphometric measurements of ONL thickness. Qualitative ISH analysis indicated that the Monarsen treatment reduced the expression of mRNA AChE-R more efficiently than it did for mRNA AChE-S (data not shown), supporting previous observations of particular vulnerability of nascent mRNA transcripts to the antisense effect. $^{27,35,36}$ These results further suggest that the N-AChE-R protein (and/or other nascent AChE variants induced under photic damage, such as N-AChE-S) play a detrimental role, exacerbating photoreceptor injury after exposure to bright, damaging light. This role can be explained in at least two alternative ways. First and foremost, the damaging role of the expressed AChE may be due to its catalytic activity. ACh has been shown to suppress the production of proinflammatory cytokines $^{40}$ and to act via nicotinic receptors as a neuroprotector against excitotoxicity. ${ }^{41,42}$ In fact, in a mammalian model of glutamate-induced excitotoxicity, ACh was found to protect GCs from death. ${ }^{43}$ Thus, overexpression of AChE by exposure to bright light may act to reduce the neuroprotection offered by endogenous ACh. In addition, recent reports show a nonenzymatic enhancement of apoptosis by AChE in cultured cells. ${ }^{19,20}$ Because retinal cell death after light exposure is by apoptosis, ${ }^{44,45}$ involving light-induced expression of AP-1, ${ }^{46}$ our findings may reflect a tissue-specific causal role of $\mathrm{N}$-AChE-R in the apoptosis of light-stressed photoreceptors. That suppression of nascent, but not the preexisting AChE, sufficed to reduce light-induced retinal damage supports the notion that distinct AChE variants play different roles in the retina. The similarity of their catalytic properties further supports the notion that nonenzymatic properties of AChE were involved.

Exposure to damaging light also induced expression of $\mathrm{N}$-AChE-R in bipolar cells and ganglion cells, raising the possibility that light-induced retinal damage is not limited to the photoreceptors, but reflects apoptotic processes in a variety of retinal neurons. Future experiments are needed to find out whether N-AChE-R accumulates in retinal (and other) neurons after other stressful episodes such as increased pressure (glaucoma), metabolic stress (diabetes), and ischemia, where it may play detrimental role(s) promoting neuronal and specifically retinal damage.

Antisense treatment has been suggested as a potential therapeutic approach in cases in which specific silencing of gene expression is desired ${ }^{47}$ However, this treatment is considered to be of limited value in the treatment of neurologic and retinal diseases because of its restricted transport across the bloodbrain barrier ${ }^{38}$ and the blood-retinal barrier (BRB). Our current findings present the photic stress-disrupted $\mathrm{BRB}^{28}$ as an exception to this rule by demonstrating that intraperitoneally injected Monarsen blocked N-AChE-R expression in the retina. That photic damage injury facilitates the functioning of Monarsen in the retina further raises the possibility of treating other retinal diseases in which the BRB is damaged (e.g., clinical and 
experimental diabetic retinopathy), ${ }^{48,49}$ by systemic antisense administration.

\section{References}

1. Stone J, Maslim J, Valter-Kocsi K, et al. Mechanisms of photoreceptor death and survival in mammalian retina. Prog Retin Eye Res. 1999;18:689-735.

2. Wenzel A, Grimm C, Samardzija M, Reme CE. Molecular mechanisms of light-induced photoreceptor apoptosis and neuroprotection for retinal degeneration. Prog Retin Eye Res. 2005;24:275-306.

3. O'Steen WK, Anderson KV, Shear CR. Photoreceptor degeneration in albino rats: dependency on age. Invest Ophthalmol. 1974;13: $334-339$.

4. LaVail MM, Gorrin GM, Repaci MA, Yasumura D. Light-induced retinal degeneration in albino mice and rats: strain and species differences. Prog Clin Biol Res. 1987;247:439-454.

5. Rapp LM, Williams TP. The role of ocular pigmentation in protecting against retinal light damage. Vision Res. 1980;20:1127-1131.

6. Barbe MF, Tytell M, Gower DJ, Welch WJ. Hyperthermia protects against light damage in the rat retina. Science. 1988;241:1817-1820.

7. LaVail MM, Unoki K, Yasumura D, et al. Multiple growth factors, cytokines, and neurotrophins rescue photoreceptors from the damaging effects of constant light. Proc Natl Acad Sci USA. 1992; 89:11249-11253.

8. Organisciak DT, Darrow RM, Jiang YI, Marak GE, Blanks JC. Protection by dimethylthiourea against retinal light damage in rats. Invest Ophthalmol Vis Sci. 1992;33:1599-1609.

9. Meshorer E, Soreq H. Virtues and woes of AChE alternative splicing in stress-related neuropathologies. Trends Neurosci. 2006;29:216-224.

10. Taylor P, Radic Z. The cholinesterases: from genes to proteins. Ann Rev Pharmacol Toxicol. 1994;34:281-320.

11. Soreq H, Seidman S. Acetylcholinesterase: new roles for an old actor. Nat Rev Neurosci. 2001;2:294-302.

12. Behra M, Cousin X, Bertrand C, et al. Acetylcholinesterase is required for neuronal and muscular development in the zebrafish embryo. Nat Neurosci. 2002;5:111-118.

13. Meshorer E, Erb C, Gazit R, et al. Alternative splicing and neuritic mRNA translocation under long-term neuronal hypersensitivity. Science. 2002;295:508-512.

14. Kaufer D, Friedman A, Seidman S, Soreq H. Acute stress facilitates long-lasting changes in cholinergic gene expression. Nature. 1998; 393:373-377.

15. Meshorer E, Bryk B, Toiber D, et al. SC35 promotes sustainable stress-induced alternative splicing of neuronal acetylcholinesterase mRNA. Mol Psychiatry. 2005;10:985-997.

16. Meshorer E, Toiber D, Zurel D, et al. Combinatorial complexity of $5^{\prime}$ alternative acetylcholinesterase transcripts and protein products. J Biol Chem. 2004;279:29740-29751.

17. Shohami E, Kaufer D, Chen Y, et al. Antisense prevention of neuronal damages following head injury in mice. $J \mathrm{Mol} \mathrm{Med}$. 2000;78:228-236.

18. Metz CN, Tracey KJ. It takes nerve to dampen inflammation. Nature Immunol. 2005;6:756-757.

19. Zhang XJ, Yang $L$, Zhao Q, et al. Induction of acetylcholinesterase expression during apoptosis in various cell types. Cell Death Differ. 2002;9:790-800.

20. Park SE, Kim ND, Yoo YH. Acetylcholinesterase plays a pivotal role in apoptosome formation. Cancer Res. 2004;64:2652-2655.

21. Bytyqi AH, Lockridge O, Duysen E, et al. Impaired formation of the inner retina in an AChE knockout mouse results in degeneration of all photoreceptors. Eur J Neurosci. 2004;20:2953-2962.

22. Broide RS, Grifman M, Loewenstein A, et al. Manipulations of ACHE gene expression suggest non-catalytic involvement of acetylcholinesterase in the functioning of mammalian photoreceptors but not in retinal degeneration. Brain Res Mol Brain Res. 1999; 71:137-148.

23. Dowling JE. The Retina: An Approachable Part of the Brain. Cambridge, MA: Harvard University Press; 1987.

24. Criswell MH, Brandon C. Acetylcholinesterase and choline acetyltransferase localization patterns do correspond in cat and rat retinas. Vision Res. 1993;33:1747-1753.

25. Hutchins JB. Acetylcholine as a neurotransmitter in the vertebrate retina. Exp Eye Res. 1987;45:1-38.
26. Karnovsky MJ, Roots L. A "direct-coloring" thiocholine method for cholinesterases. J Histochem Cytochem. 1964;12:219-221.

27. Brenner T, Hamra-Amitay Y, Evron T, et al. The role of readthrough acetylcholinesterase in the pathophysiology of myasthenia gravis. FASEB J. 2003;17:214-222.

28. van Best JA, Putting BJ, Oosterhuis JA, Zweypfenning RC, Vrensen GF. Function and morphology of the retinal pigment epithelium after light-induced damage. Micro Res Technol. 1997;36:77-88.

29. Li Q, Zemel E, Miller B, Perlman I. Early retinal damage in experimental diabetes: electroretinographical and morphological observations. Exp Eye Res. 2002;74:615-625.

30. Armington JC. The Electroretinogram. New York: Academic Press; 1974.

31. Lei B, Perlman I. The contribution of voltage- and time-dependent potassium conductances to the electroretinogram in rabbits. Vis Neurosci. 1999;16:743-754.

32. Ripps H, Witkovsky $P$. Neuron-glia interaction in the brain and retina. Prog Retinal Res. 1985;4:181-219.

33. Fulton AB, Hansen RM. Scotopic stimulus/response relations of the b-wave of the electroretinogram. Doc Opbthalmol. 1988;68:193304.

34. Hood DC, Birch DG. A computational model of the amplitude and implicit time of the b-wave of the human ERG. Vis Neurosci. 1992;8:107-126.

35. Evron T, Ben-Moyal L, Lam N, Soreq H. RNA-targeted suppression of stress-induced allostasis in monkey spinal cord neurons. Neurodegen Dis. 2005;2:16-27.

36. Nijholt I, Farchi N, Kye M, et al. Stress-induced alternative splicing of acetylcholinesterase results in enhanced fear memory and longterm potentiation. Mol Psychiatry. 2004;9:174-183.

37. Cohen O, Erb C, Ginzberg D, et al. Neuronal overexpression of 'readthrough' acetylcholinesterase is associated with antisensesuppressible behavioral impairments. Mol Psychiatry. 2002;7: $874-885$

38. Soreq H, Seidman S. Antisense approach to isoform-specific blockade of acetylcholinesterase. In: Crooke ST, ed. Antisense Drug Technology: Principles, Strategies and Applications. Carlsbad, CA: Marcel Dekker, Inc.; 2001:565-586.

39. Rapp LM, Naash MI, Wiegand RD, et al. Morphological and biochemical comparison between retinal regions having differing susceptibility to photoreceptor degeneration. In: LaVail MM, Hollyfield JG, Anderson RE, eds. Retinal Degeneration: Experimental and Clinical Studies. New York: Alan R. Liss; 1985:421-437.

40. Pavlov VA, Tracey KJ. The cholinergic anti-inflammatory pathway. Brain Behav Immun. 2005;19:493-499.

41. Stevens TR, Krueger SR, Fitzsimonds RM, Picciotto MR. Neuroprotection by nicotine in mouse primary cortical cultures involves activation of calcineurin and L-type calcium channel inactivation. J Neurosci. 2003;23:10093-10099.

42. Hejmadi MV, Dajas-Bailador F, Barns SM, Jones B, Wonnacott S. Neuroprotection by nicotine against hypoxia-induced apoptosis in cortical cultures involves activation of multiple nicotinic acetylcholine receptor subtypes. Mol Cell Neurosci. 2003;24:779-786.

43. Wehrwein E, Thompson SA, Coulibaly SF, Linn DM, Linn CL. Acetylcholine protection of adult pig retinal ganglion cells from glutamate-induced excitotoxicity. Invest Opbthalmol Vis Sci. 2004; $45: 1531-1543$.

44. Hafezi F, Marti A, Munz K, Reme CE. Light-induced apoptosis: differential timing in the retina and pigment epithelium. Exp Eye Res. 1997;64:963-970.

45. Roca A, Shin K-J, Liu X, Simon MI, Chen J. Comparative analysis of transcriptional profiles between two apoptotic pathways of lightinduced retinal degeneration. Neuroscience. 2004;129:779-790.

46. Grimm C, Andreas W, Hafezi F, Reme CE. Gene expression in the mouse retina: the effect of damaging light. Mol Vis. 2000;6:252260.

47. Opalinska JB, Gewirtz AM. Therapeutic potential of antisense nucleic acid molecules. Sci STKE. 2003;206:1-5.

48. Cunha-Vaz JG. Studies on the pathophysiology of diabetic retinopathy: the blood-retinal barrier in diabetes. Diabetes. 1983; $32: 20-27$.

49. Xu Q, Qaum T, Adamis AP. Sensitive blood-retinal barrier breakdown quantitation using Evans blue. Invest Ophthalmol Vis Sci. 2001;42:789-794. 